\title{
Eficácia do florfenicol e da oxitetraciclina no controle de Aeromonas hydrophila em pacu (Piaractus mesopotamicus)
}

[Efficacy of the florfenicol and of the oxytetracycline in the control in Aeromonas hydrophila in pacu (Piaractus mesopotamicus)]

\author{
S.P. Carraschi ${ }^{1,2}$, C. Cruz $^{2}$, J.G. Machado Neto ${ }^{3}$, M.P. Castro ${ }^{4}$, N.L. Bortoluzzi ${ }^{4}$, A.C.F. Gírio ${ }^{2}$ \\ ${ }^{1}$ Centro de Aquicultura da Unesp - Campus de Jaboticabal - Jaboticabal, SP \\ ${ }^{2}$ Núcleo de Estudos e Pesquisas Ambientais em Matologia - NEPEAM \\ Faculdade de Ciências Agrárias e Veterinárias \\ Via de Acesso Prof. Dr. Paulo Donato Castellani, s/ nº Zona Rural \\ 14884-900 - Jaboticabal, SP \\ ${ }^{3}$ Laboratório de Ecotoxicologia dos Agrotóxicos e Saúde Ocupacional (Laborseg) \\ Faculdade de Ciências Agrárias e Veterinárias - Unesp de Jaboticabal - Jaboticabal, SP \\ ${ }^{4}$ Laboratório de Ictiopatologia - Faculdade de Ciências Agrárias e Veterinárias - Jaboticabal, SP
}

\section{RESUMO}

Determinou-se a concentração eficaz de oxitetraciclina (OTC) e florfenicol (FFC) no tratamento de Aeromonas hydrophila em pacu (Piaractus. mesopotamicus). Os pacus foram submetidos à captura duas vezes ao dia por quatro dias e em seguida foram infectados com $A$. hydrophila $\left(2,4 \times 10^{7}\right.$ bactéria $\left.\mathrm{mL}^{-1}\right)$. Os tratamentos utilizados foram: controle sem infecção (CSI), controle com infecção (CCI) e tratados com 110,0; 140,0 e 170,0mgOTC. $\mathrm{kg}^{-1}$, e 5,0; 10,0 e 15,0mgFFC. $\mathrm{kg}^{-1}$. As variáveis de qualidade da água foram monitoradas diariamente. Após o tratamento, no CSI dos dois testes, ocorreu 100\% de sobrevivência. Nos testes com OTC, no CCI, a sobrevivência foi de 29,2\%; em 110,0mg. $\mathrm{kg}^{-1}, 37,5 \%$; em 140,0mg. $\mathrm{kg}^{-1}$, $29,2 \%$; e em $170,0 \mathrm{mg} \mathrm{kg}^{-1}, 50,0 \%$. Nos testes com FFC, foi eficaz com $10,0 \mathrm{mg}^{-\mathrm{kg}^{-1}}$, e no CCI a sobrevivência foi de 76,9\%; em 5,0mg.kg-1, 81,81\%; em 10,0mg/L.kg ${ }^{-1}, 100 \%$ e em 15,0mg.kg-1 $, 87,5 \%$. A OTC, em concentrações de até $170,0 \mathrm{mg} \cdot \mathrm{kg}^{-1}$ de ração, não é eficaz para o controle de A. hydrophila em pacu, e o FFC é eficaz na concentração de $10,0 \mathrm{mg} \mathrm{kg}^{-1}$ e ambos não alteram as variáveis de qualidade de água.

Palavras-chave: tratamento, doenças, bactérias, antibióticos

\begin{abstract}
The effective concentration of antibiotics OTC and FFC in the treatment of Aeromonas hydrophila in раси (Piaractus mesopotamicus). The pacus were subjected to capture twice daily (four days) and then were infected with A. hydrophila $\left(2.4 \times 10^{7}\right.$ bacteria $\left.\mathrm{mL}^{-1}\right)$. The treatments were: control without infection (CSI), with infection control (CCI) and 110.0, 140.0 and 170.0mgOTC. $\mathrm{kg}^{-1}$, and 5.0, 10.0 and $15.0 \mathrm{mgFFC.} \mathrm{kg}^{-1}$. The variables of water quality were monitored daily. After treatment, the CSI of the two experiments was $100 \%$ survival. In tests with OTC, the CCI was $29.17 \%$, in $110.0 \mathrm{mg} . \mathrm{kg}^{-1}$, $37.5 \%$, in $140.0 \mathrm{mg} \cdot \mathrm{kg}^{-1}, 29.17 \%$ and in $170.0 \mathrm{mg} \cdot \mathrm{kg}^{-1}, 50.0 \%$. The FFC was effective with $10.0 \mathrm{mg} \mathrm{kg}^{-1}$, and in the CCI the survival was $76.9 \%$, in $5.0 \mathrm{mg} \cdot \mathrm{kg}^{-1}, 81.81 \%$, in $10.0 \mathrm{mg} / \mathrm{L} . \mathrm{kg}^{-1}, 100 \%$ and in $15.0 \mathrm{mg} . \mathrm{kg}^{-1}, 87.5 \%$. The OTC in concentrations of up to $170.0 \mathrm{mg} . \mathrm{kg}^{-1}$ of ration is not effective in the control of A. hydrophila in pacu and the FFC is effective in the concentration of $10.0 \mathrm{mg} \mathrm{kg}^{-1}$ and this antibiotic does not change the variables of water quality.
\end{abstract}

Keywords: treatment, diseases, bacteria, antibiotics

Recebido em 19 de maio de 2010

Aceito em 6 de abril de 2011

E-mail: pacarraschi@yahoo.com.br 


\section{Carraschi et al.}

\section{INTRODUÇÃO}

As bacterioses destacam-se como importantes fatores limitadores da produtividade, pois provocam atraso no crescimento e alta taxa de mortalidade (Ranzani-Paiva et al., 1997), e são consideradas um dos principais problemas da aquicultura em sistemas de cultivo intensivo. As bactérias do gênero Aeromonas estão entre os patógenos que mais causam perdas econômicas, sendo responsáveis por grande parte das doenças dos animais de cultivo. Elas são encontradas em diversos habitats e as espécies que causam doenças em peixes são: $A$. hydrophila, $A$. veronii, A. sobri, A. cavie, A. allosaccharophila e A. salmonicida (Austin e Austin, 1987). A A. hydrophila, um bastonete móvel gram-negativo, causa septicemia hemorrágica em peixes, caracterizada por apresentar lesões superficiais e hemorragias focais como petéquias, e exoftalmia. Internamente, pode haver acúmulo de líquido ascítico, anemia e lesões no fígado e rins, como pontos de necrose (Austin e Austin, 1987).

Entre os antibióticos, a oxitetraciclina (OTC) e o florfenicol (FFC) são os mais utilizados para tratar infecções causadas por bactérias gramnegativas em peixes (Rigos e Troisi, 2005). A OTC apresenta amplo espectro de ação contra os patógenos de peixes e baixa toxicidade (Barragy, 1994) e é eficaz no tratamento de colunariose e aeromonose (Bullock et al., 1986; Thomas-Jinu e Goodwin, 2004; Andrade et al., 2006). O FFC apresenta eficácia nas infecções por $A$. salmonicida subsp. salmonicida, A. hydrophyla, F. psychrophilum, Yersinia ruckeri e Vibrio anguillarum (Fukui et al., 1987; Nordmo et al., 2006).

Este trabalho teve como objetivos determinar a concentração dos antibióticos oxitetraciclina $\left(\right.$ Terramicina $^{\circledR}$ ) e florfenicol (Aquaflor ${ }^{\circledR}$ ) no tratamento da infecção experimental por $A$. hydrophila em pacu e avaliar as variáveis de qualidade da água durante o tratamento com esses xenobióticos.

\section{MATERIAL E MÉTODOS}

Pacus, com média de peso de $33,08 \pm 4,97$ gramas, foram mantidos em caixa de 400 litros, e duas vezes ao dia, durante quatro dias, foram submetidos à captura. Os peixes foram retirados da água por dois minutos como estímulo estressante como forma de causar imunossupressão (Urbinati et al., 2004). Após este período de indução de estresse, foram infectados com A. hydrophila por injeção intracelomática de $2,4 \times 10^{7}$ bactéria $\mathrm{mL}^{-1}$ (escala McFarland). Vinte e quatro horas após a infecção foram observados os sinais clínicos causados pela bactéria, como: dilatação do celoma, exoftalmia, lesões cutâneas bilaterais avermelhadas com erosão da pele e corrosão da nadadeira caudal, após o que se iniciou o tratamento com os antibióticos.

Foram realizados dois experimentos, um para cada antibiótico. No experimento com OTC, foram utilizados 104 peixes, e com FFC 64 peixes. Os tratamentos utilizados foram: controle sem infecção (CSI) - peixes alimentados com ração comercial -, controle com infecção (CCI) - peixes alimentados com ração comercial - e três concentrações de cada antibiótico - peixes alimentados com ração comercial -, com três repetições por tratamento e em delineamento inteiramente ao acaso. Os antibióticos utilizados foram a Terramicina ${ }^{\circledR}$, com 5,5gOTC. $100 \mathrm{~g}^{-1}$, e o Aquaflor $^{\circledR}$, com 500gFFC.kg-1 . Para os grupos tratados com antibióticos, foi utilizada a ração da Poli Nutri (32\% PB) e em cada quilo de ração foram acrescidos, de forma homogênea, 110,0; 140,0 e $170,0 \mathrm{mg}$ de OTC. $\mathrm{kg}^{-1}$ ou 5,$0 ; 10,0$ e 15,0mg de FFC. $\mathrm{kg}^{-1}$ dissolvidos em $2 \%$ de óleo vegetal. Essa ração foi mantida em temperatura ambiente por quatro dias para secagem. O tratamento com OTC teve duração de sete dias, e o com FFC 10 dias, à taxa de $2,0 \%$ do peso corporal ao dia e manutenção do fluxo de água constante de $9,0 \mathrm{ml} . \mathrm{s}^{-1}$.

O antibiótico foi considerado eficaz quando apresentou, no mínimo, 90\% de eficácia, de acordo com a Portaria do Ministério da Agricultura, Pecuária e Abastecimento do Brasil n. 48 (Brasil, 1997), relativo a parasitos, adaptada para bactérias.

As condições iniciais das variáveis de qualidade da água foram: temperatura $25 \pm 2^{\circ} \mathrm{C}$; $\mathrm{pH}$ entre 6,5 e 7,5; $\mathrm{O}_{2} \mathrm{D}$ entre 7,0 e $8,0 \mathrm{mgL}^{-1}$ e condutividade elétrica entre 180,0 e $185,0 \mathrm{mScm}^{-1}$, e foram monitoradas diariamente durante o tratamento, duas vezes ao dia (manhã e tarde). 
Esse projeto foi aceito pelo Comitê de Ética da Faculdade de Ciências Agrárias e Veterinárias da Unesp de Jaboticabal, protocolo n ${ }^{0}$ 028409-08.

\section{RESULTADOS}

No experimento com OTC, a taxa de sobrevivência no CSI foi de $100 \%$. A OTC não foi eficaz no controle da infecção causada por $A$. hydrophila. Após sete dias de alimentação no CCI, a taxa de sobrevivência foi de 29,2\%; em 110,0mg. $\mathrm{kg}^{-1}$, 37,5\%; em 140,0mg.kg ${ }^{-1}$, 29,2\% e em $170,0 \mathrm{mg}^{\mathrm{kg}}{ }^{-1}, \quad 50,0 \%$. Do total de 104 organismos que iniciaram o experimento com OTC somente 43 sobreviveram após sete dias de tratamento, mesmo se considerando os peixes do CCI e os tratados.

Uma hora após o arraçoamento com OTC, ainda se observava sobra de alimento, indicando baixo consumo de ração.

No experimento com FFC, a taxa de sobrevivência no CSI foi também de 100\%. Após 10 dias de alimentação no CCI, a taxa de sobrevivência foi de $76,9 \%$; em $5,0 \mathrm{mg} \cdot \mathrm{kg}^{-1}$, 81,8\%; em $10,0 \mathrm{mg} / \mathrm{L}_{\mathrm{kg}}{ }^{-1}, \quad 100 \%$; e em $15,0 \mathrm{mg} \cdot \mathrm{kg}^{-1}, 87,5 \%$. Do total de 64 peixes que iniciaram o experimento com FFC, 57 permaneceram até o final de 10 dias.

No tratamento com FFC, somente no primeiro e no segundo dia de alimentação, ocorreu pequena sobra de ração. Após esse período, o consumo foi total (2,0\% do peso corporal).

Após o tratamento com os dois antibióticos, ocorreu melhora dos sinais clínicos, com cicatrização da epiderme. Alguns sinais, como o avermelhamento da periferia da ferida, e algumas lesões com erosão da pele ainda estavam presentes em alguns animais do CCI e nos do tratamento com OTC. Isso pode ser atribuído ao fato de que esse antibiótico não foi eficaz no controle de A. hydrophila e o CCI não ocorreu medicação; assim, as lesões não apresentaram total cicatrização.

As variáveis de qualidade de água não foram alteradas durante o tratamento com os antibióticos em nenhum dos períodos de avaliação (manhã e tarde), estando dentro dos parâmetros iniciais dos experimentos.

\section{DISCUSSÃO}

O pequeno consumo de ração nos tratamentos com OTC sugere baixa palatabilidade. Rigos et al. (1999), ao trabalharem com ração adicionada OTC com 5\% de óleo, também observaram baixa palatabilidade para Dicentrarchus labrax. Segundo esses autores, a palatabilidade é maior quando a OTC é incluída durante a preparação da ração, o que poderia aumentar a eficácia desse antibiótico.

A OTC foi $100 \%$ eficaz para outras espécies, incluindo para Salvelinus fontinalis para tratar úlcera (Snieszko et al., 1951); para I. punctatus para tratar aeromonose, com 50,0mg. $\mathrm{kg}^{-1}$ (Oxytetracycline, 1986) e em I. punctatus no tratamento de F. columnare, com $80,0 \mathrm{mg} \cdot \mathrm{kg}^{-1}$ (Tomas-Jinu e Goodwin, 2004). Porém, neste trabalho a maior eficácia observada foi de $50 \%$ em 170,0mg. $\mathrm{kg}^{-1}$. Talvez essa baixa eficácia possa ser pelo efeito da infecção, pela perda de apetite ou pela baixa palatabilidade da ração.

As concentrações recomendadas encontradas na literatura estão entre 50,0mg. $\mathrm{kg}^{-1}$ e $1750 \mathrm{mg} \cdot \mathrm{kg}^{-1}$ (Scott, 1993; Namdari et al., 1996; Aquaculture..., 1998; Ueno et al., 2004; Wang e Li, 2004), dependendo da bactéria, da espécie de peixe e das variáveis físico químicas da água. Mesmo se utilizando concentrações entre esse intervalo de concentração, não foi obtida eficácia de controle de infecção experimental de $A$. hydrophila em pacu.

A concentração de 5,0mg de FFC não foi suficiente para melhorar o estado debilitado dos peixes infectados com $A$. hydrophila e refazer a homeostasia (Darwish, 2007). A de 10,0mg de FFC foi suficiente para promover a homeostasia, apresentando $100 \%$ de eficácia, ou seja, nenhum peixe morreu (Aquaflor, 2010b). Já 15,0mg de FFC foi uma concentração muito alta, sendo que o antibiótico começa a competir pelo sítio metabólico e não consegue reestabelecer o seu estado saudável por despender muita energia para metabolizar o antibiótico.

A sobrevivência foi maior em pacu tratado com 10,0mg. $\mathrm{kg}^{-1}$ (100\%) do que em salmão para tratar A. salmonicida (87\%) (Inglis et al., 1991). Para o controle de Streptococcus iniae em Morone chrysops x $M$. saxatilis, a eficácia dessa mesma concentração também foi menor $(69,2 \%)$ do que 
para o pacu; porém, com 5,0mg. $\mathrm{kg}^{-1}$ e $15,0 \mathrm{mg} \cdot \mathrm{kg}^{-}$ 1 , a eficácia foi maior $(86,7 \%$ e $94,2 \%$, respectivamente) do que para o tratamento de aeromonose em pacu (Darwish, 2007), indicando que a concentração eficaz de tratamento de $S$. iniae está entre 10,0 e $15,0 \mathrm{mg} \cdot \mathrm{kg}^{-1}$, enquanto, para o pacu, foi de $10 \mathrm{mg} \cdot \mathrm{kg}^{-1}$.

A concentração eficaz de $10,0 \mathrm{mg} \cdot \mathrm{kg}^{-1}$ de FFC está de acordo com a indicada para I. punctatus para tratar E. ictaluri (Gaikowski et al., 2003; Aquaflor, 2010b); para salmão para tratar furunculoses (Inglis et al., 1991; Nordmo et al., 2006); para G. morhua para tratar vibriose (Seljestokken et al., 2006) e para O. niloticus para tratar A. hydrophila (Aquaflor, 2010a).

\section{CONCLUSÃO}

A OTC, em concentrações de até $170,0 \mathrm{mg} \cdot \mathrm{kg}^{-1}$ de ração, não é eficaz para o controle de $A$. hydrophila em pacu, e o FFC é eficaz na concentração de $10,0 \mathrm{mg} \cdot \mathrm{kg}^{-1}$ para o controle e o tratamento de infecção por $A$. hydrophila em pacu. O uso desses antibióticos não alterou as variáveis de qualidade de água.

\section{AGRADECIMENTOS}

À Fundação de Amparo a Pesquisa do Estado de São Paulo (Fapesp), pelo auxílio ao projeto de pesquisa, proc. $n^{0}$ 2008/51900-3, e ao Conselho Nacional de Desenvolvimento Científico e Tecnológico (CNPq), pela bolsa concedida, proc. nº 136523/2009-9.

\section{REFERÊNCIAS BIBLIOGRÁFICAS}

ANDRADE, L.S.; ANDRADE, R.L.B.; BECKER, A.G. et al. Survival and behavior of silver catfish, Rhamdia quelen, submitted to antibiotics and sodium chloride treatments. Cienc. Rural, v.36, p.1004-1007, 2006.

AQUACULTURE Drugs.” In: Fish and fishery products hazards and controls guide. (2.ed.). FDA: Washington: FDA,1998. Cap.11, p.115-132.

AQUAFLOR. Florfenicol. Technical Monograph for catfish health professionals. Schering-Plough Animal Health, 2010a 2p. Disponível em: http://www.aquaflor-usa.com/pdfs/Catfish_Brochure.pdf. Acessado em: 2 dez. 2009.
AQUAFLOR. Florfenicol. Technical Monograph for catfish health professionals. Schering-Plough Animal Health, 2010a 36p. Disponível em: http://www.aquaflor-usa.com/pdfs/Catfish_Brochure.pdf. Acessado em: 28 nov. 2009.

AUSTIN, B.; AUSTIN, D.A. Bacterial fish pathogens: disease in farmed and wild fish. Chichester: Ellis Horwood Limit, 1987. 171-173p.

BARRAGY, T.B. (Ed.). Veterinary drug therapy Malvern, USA: Lea \& Febinger, 1994. 264-275p.

BRASIL. Ministério da Agricultura, Pecúaria e Abastecimento. Portaria, n. 48, de 12 de maio de 1997. Regulamento técnico para licenciamento e/ou renovação de licença de produtos antiparasitários de uso veterinário. p.15, 1997.

BULLOCK, G.L.; HSU, T.C.; SHOTTS, E.B.J.R. Columnaris disease of fishes. Washington: Fish and Wildlife Service Fish Disease Leaflet, 1986. p.9.

DARWISH, A.M. Laboratory efficacy of florfenicol against Streptococcus iniae infection in sunshine bass. J. Aquat. Anim. Health, v.19, p.1-7, 2007.

FUKUI, H.; FUJIHARA, Y.; KANO, T. In vitro and in vivo antibacterial activities of florfenicol, a new fluorinated analog of thiamphenicol, against fish pathogens. Fish Pathol., v.22, p.201-207, 1987.

GAIKOWSKI, M.P.; WOLF, J.C.; ENDRIS, R.G.; GINGERICH, W.H. Safety of aquaflor (florfenicol, $50 \%$ type a medicated article), administered in feed to channel catfish, Ictalurus punctatus. Toxicol. Pathol., v.3, p.689-697, 2003.

INGLIS, V.; RICHARDS, R.H.; VARMA, K.J. et al. Florfenicol in Atlantic salmon, Salmo salar L, parr: tolerance and assessment of efficacy against furunculosis. J. Fish Dis., v.14, p.343-351, 1991.

NAMDARI, R.; ABEDINI, S.; LAW, F.C.P. Tissue distribution and elimination of oxytetracycline in seawater chinook and salmon following medicatedfees treatment. Aquaculture, v.144, p.27-38, 1996. NORDMO, R.; VARMA, K.J.; SUTHERLAND, I.H. et al. Florfenicol in Atlantic salmon, Salmon salar L.: field evaluation of efficacy against furunculosis in Norway. J. Fish Dis., v. 17, p.239244, 2006.

OXYTETRACYCLINE. In: Code of Federal regulations. Washington, DC: U.S. Government Printing Office, 1986. p.586-589. 
RANZANI-PAIVA, M.J.T.; ISHIKAWA, C.M.; CAMPOS, B.E.S. et al. Hematological characteristics associated with parasitism in mullets, Mugil platanus, from the estuarine region of Cananéia, São Paulo, Brasil. Rev. Bras. Zool., v.14, p.329-339, 1997.

RIGOS, G.; ALEXIS, M.; NENGAS, I. Leaching, palatability and digestibility of oxolinic acid and oxytetracycline in sea bass (Dicentrarchus labrax L.) Aquac. Res., v.30, p.1-7, 1999.

RIGOS, G.; TROISI, G.M. Antibacterial agents in Maditerranean finfish farming: A sinopsis of drug pharmacokinetics in important euryhaline fish species and possible environmental implications. Rev. Fish Biol. Fish, v.15, p.53, 2005.

SCOTT, P. Therapy in aquaculture. In: BROWN, L. (Ed.), Aquaculture for veterinarians. Oxford: Pergamon Press, 1993. 131-153p.

SELJESTOKKEN, B.; BERGH, O.; MELINGEN, G.O.; RUDRA, H. et al. Treating experimentally induced vibriosis (Listonella anguillarum) in cod, Gadus morhua L., with florfenicol. J. Fish Diseases, v.29, p.737-742, 2006.
SNIESZKO, S,F.; FRIDDLE, S.B.; GRIFFIN, P.J. Successful treatment of ulcer disease in brook trout (Salvelinus fontinalis) with terramycin. Sci, v.113, p.2, 1951.

THOMAS-JINU, D.C.S.; GOODWIN, A.E. Acute columnaris infection in channel catfish, Ictalurus punctatus (Rafinesque): efficacy of practical treatments for warmwater aquaculture ponds. $J$. Fish Dis., v.27, p.23-28, 2004.

UENO, R.; KINOSHITA, A.; WAKABAYASHI, J. Comparative pharmacokinetics of oxytetracycline in eel and its fate in a closed aquatic environment. Aquaculture, v.235, p.53-63, 2004.

URBINATI, E.C.; ABREU, J.S.; CAMARGO, A.C.S. et al. Loading and transport stress of juvenile matrinxã (Brycon cephalus, Characidae) at various densities. Aquaculture, v.229, p.389-400, 2004.

WANG, Q.; LI, J. Tissue distribution and elimination of oxytetracycline in perch Lateolabras japonicus and black seabream (Sparus macrocephalus) following oral administration. Aquaculture, v.237, p.31-40, 2004. 